\title{
Influence of External Opinions on an Individual Choice in Conditions of Uncertainty: Experiment and Laser-like Model
}

\author{
Nikita Solovyev ${ }^{1}$, Dmitrii Gorbatov ${ }^{2}$, Leonid Soms ${ }^{3, *}$ \\ ${ }^{1}$ Faculty of Applied Psychology, St. Petersburg State Institute of Psychology and Social Work, St. Petersburg, Russia \\ ${ }^{2}$ Department of Management of Mass Communications, St. Petersburg State University, St. Petersburg, Russia \\ ${ }^{3}$ Centre of Scientific and Educational Publications and Conferences, University ITMO, St. Petersburg, Russia \\ Email address: \\ leonid.soms@gmail.com (L. Soms) \\ *Corresponding author

\section{To cite this article:} \\ Nikita Solovyev, Dmitrii Gorbatov, Leonid Soms. Influence of External Opinions on an Individual Choice in Conditions of Uncertainty: \\ Experiment and Laser-like Model. American Journal of Psychiatry and Neuroscience Vol. 8, No. 1, 2020, pp. 12-17. \\ doi: 10.11648/j.ajpn.20200801.13
}

Received: December 9, 2019; Accepted: January 15, 2020; Published: February 4, 2020

\begin{abstract}
A psychological experiment for quantitative evaluation of degree of external opinions influencing the choice of a participant in conditions of uncertainty was suggested. A computer-driven experiment was performed, with 256 participants. In the experiment participating volunteers were shown a series of images accompanied by two not-evident alternative answers for a question "what is this?", and were prompted to make their choice under different condition. These conditions were: (i) a free individual choice (control group); (ii) a choice when each participant sees the results of real answers of other subjects of his experimental group ("true" group); (iii) a choice when the participants see false response promptings generated by a special computer program ("false" groups). Quantitative results were obtained showing an influence of "psychological pressure" to a frequency of occurrence of alternative answers. A laser-like model is presented for energy levels in a brain and for decision making in such conditions. The laser-like model is based on a usual system of kinetic equations describing dependence of population of excited and ground levels (before and after the answer, respectively), and the number of different answers (zero known answers for control group, number of real or manipulated answers for "true" and "false" groups). By fitting parameters of the model to results of the experiment, numerical values of parameters of the model ("coefficients of stimulating influence" analogues to Einstein coefficients $B_{i k}$ for stimulated emission of radiation) were determined.
\end{abstract}

Keywords: Peer Group Pressure, Freedom of Choice, Manipulated Information, Laser-like Model

\section{Introduction}

Recently, quasi-physical approaches are actively developed in application for psychological and social problems. A particular interest is attracted to a problem of choice under conditions of uncertainty; deviations from classical paradigm based on logic and statistics were noticed. Thus, a violation of the conjunction law was found [1] at answers of subjects for sequential range of questions, such violation being impossible to explain within classical theory of probability. An attempt of solution led to an alternative interpretation based on the quantum logic [2]. The essence of this interpretation was that a certain vector of state was assigned to human's state of mind for the moment of making the answer, this vector representing a superposition of two alternative answers. After the decision of a definite answer is made, the vector of state of the mind collapsed into one of two possible final states.

Studies of quantum or quantum-like effects in a human behavior and functioning of a brain are important not only for practice but also for understanding phenomena of consciousness and free will [3-7] which have no generally recognized explanation nor in science neither in philosophy.

In connection with this, examination of analogies between lasers as macroscopic objects with quantum behavior, from one side, and complex biological [8] and social [9] systems, from the other side, could be productive. 
In spite of the fact that ideas of quantum- and laser-like features of psychological and social behavior exist for a relatively long time, until present there are nor their experimental evidence neither theoretical models corresponding to a laser specificity of a decision making by several interacting individuals.

The goals of our study were:

1. to develop the scheme of a psychological experiment for quantitative evaluation of degree of external opinions influencing the choice of a participant in conditions of uncertainty, and to perform the experiment;

2. to formulate a laser-like model of the experiment;

3. to determine, by fitting parameters of the model to results of the experiment, empirical numerical values of parameters of the model.

The presented work describes results of an experiment of computer testing in which participants were shown a series of images accompanied by two not-evident alternative answers for a question "what is this?", and were prompted to make their choice under different conditions, viz.:

i. A free individual choice ("control" group);

ii. A choice when each participant sees the results of real answers (response promptings) of other subjects of his experimental group ("true" group);

iii. A choice when the participants see false response promptings generated by a special computer program ("false" groups).

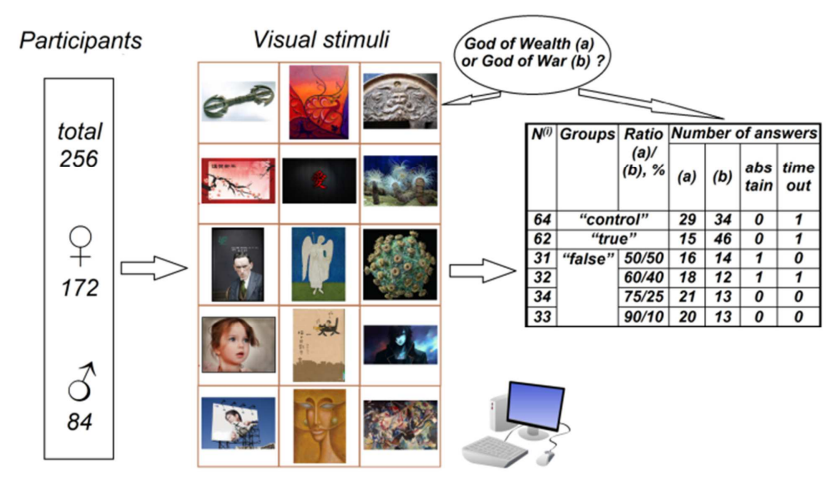

Figure 1. General scheme of experiment.

A quasi-physical model was developed in which the process of choice was presented as an aggregation of spontaneous and stimulated transitions of the state of brain from "excited" into one of alternative "lower" states.

\section{Experiment}

\subsection{Participants}

A general scheme of the experiment is shown in Figure 1.

A group of 265 volunteers (bachelor students of $1^{\text {st }}-4^{\text {th }}$ years of St. Petersburg State Institute of Psychology and Social Work), including 84 male youths and 172 girls, was divided into 6 groups and was subjected to a computercontrolled test.

In order to minimize an influence of sex and age of the participants to the results of the experiment, each group included persons of both sexes proportional to their relative part in the whole set of participants, and consisted of approximately even number of students from junior and senior classes.

A standard computer class of the Institute was used for the experiment. Each session of the experiment was in the end of the 4 th of 6 th hour of routine education process and lasted for 16-17 minutes.

The number of participants in each session was limited by the number of computers in the classroom and varied from 14 to 20 persons.

The numbers of participants in each group $N(i)$ ("control", "true", and four "false" groups) are shown in Figure 1.

\subsection{Software}

Specialized proprietary software was developed and used in the experiment. The software was designed as a WEBapplication with necessary stack of technological instruments.

Front-end - HTML5, CSS3, JavaScript, jQuery library. Back-end: - PHP5. Data manager - MySQL. The WebSocket technology was introduced for global synchronization with HTTP protocol.

The software complex includes three blocks:

1). Operator control panel: to create, edit, load/unload the tasks; to control the WebSocket server; to set time intervals for answers and number of participants.

This block provided also varying the regime of experiment:

(a) free individual choice (for the "control" goup);

(b) response promptings by demonstration of real answers of other participants of the group ("true group");

(c) response promptings by demonstrating false answers of other participants of the group, with predetermined arbitrary frequency of occurrence of alternative answers (four "false" groups).

The answers and their statistical characteristics were displayed. An algorithm of generation false promptings was as follows: just after the moment of answer of a participant, a generated false response prompting appeared on displays of all other participants. The frequencies of appearance of the false promptings to alternative answers $N_{a} / N_{b}$, for different groups in our experiment, were equal to $50 / 50,60 / 40,75 / 25$, 90/10.

2) User interface (the sequential presentation of visual stimuli and questions to be answered, with of without real or false response promptings).

3) WebSocket-server - provided the interactive real-time exchange of information.

\subsection{Stimuli}

In the course of the experiment, 15 cognitiveundetermined tasks were used. Each task included a picture, a question, and two alternative answers (a) and (b) for the question. A participant could: (i) to choose one of the answers; (ii) to abstain from the answer (push an "answer" 
button without choosing); (iii) not to answer during 40 seconds given for each stimulus. In order to eliminate or minimize a possible influence of the order of presentation of stimuli and the order of suggested answers (a) or (b), the order of presentation of stimuli and of the answers changed from group to group.

Examples of several stimuli and alternative answers are shown in Figure 2.

The information about response promptings was represented as a colored strip (red for (a) and blue for (b)) in the lower part of the screen, the length of the strips showed the number of corresponding answers. Besides, the relation of $(a) /(b)$ in percents was shown.

\subsection{Instructions and motivation of participants}

The participants were warned that the test does not pursues a)

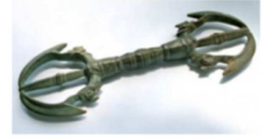

(a) a divining rod (b) a weapon

c)

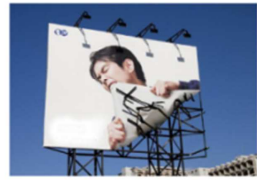

(a) an ad of medicine

(b) an ad of movie b)

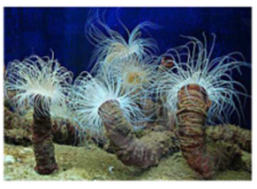

(a) an animal

(b) a plant

d)

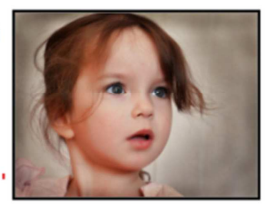

(a) a surprise

(b) a fear
Figure 2. Examples of visual stimuli and alternative answers.

an evaluation of erudition or intellectual capacity of a person and that it has no relation to teaching process. It was noted that the registration of the answers is "blind", no personal identification is applied.

In order to hide the independent variable of the study, the participants were told about a false target of the study as a reveal of influence of a type of cognitive uncertainty on the success of the task fulfillment.

The participants were instructed that the time allocated for each task is equal to 40 seconds and the rules of answering, as described above. The participants were informed that the evasion from an answer is not desirable since it makes the interpretation of the experimental results difficult.

The motivation was limited to a reference to "results of previous studies which have shown that the bachelor students of our institute were as good in solution of cognitiveundetermined tasks as masters of a prestigious university".

The first task in the series (see Figure 2a) was presented to participants as a training, with the comments of the experimenter as follows:"So, what do you see at the picture? Consider what is a material from which the thing is manufactured? What could be the age of the thing?

If this is a weapon is it a contact or it can act distantly?
How could a warrior held it? How he could use it against an enemy?

If it is divining item, to what cult - polytheistic or monotheistic could it relate? Does it bear some esoteric symbols? How could it be used by a priest?

Please do not discuss the task with other participants and make your personal choice.

As for the next pictures, make your analysis and choice on your own".

Talks and discussions were told as not desirable.

Often, an expression of bewilderment aroused, which were parried by reference to a specificity of cognitiveundetermined tasks and advises were given to examine the picture more thoroughly.

The input briefing and registration of the group took not more than six minutes, and the further execution of the tasks - about ten minutes. No final debriefing was provided.

\section{Results of Experiment}

As the result, for each visual stimulus the experimenter got a set of answers, an example of the set is given in the table in Figure 1.

The total fraction of abstained and refused answers was $11 \%$, and these cases were excluded from further calculations.

The ratio of frequencies of occurrence of alternative answers (a) and (b) $K=N a / N_{b}$ obtained for the "control" group represents the degree of equivalence of the answers. For all set of stimuli, the absolute value of deviation of $K$ from unity $|\Delta|=|K-1|$ varied from $|\Delta|_{\max }=2,5$ (for the stimulus $\# 1$, Figure 2a) to $|\Delta|_{\min }=0,05$ (for the stimulus \#7, the second row in the middle column, Figure 1; the task for this stimulus was: what means this hieroglyph: (a) a luck, (b) a sorrow), and for $70 \%$ of stimuli this value $|\Delta|_{\min }<0,5$.

At analysis, Pearson's chi-squared $\chi^{2}$ - test was applied: differences between data distribution are considered as statistically valid if empirical value $\chi^{2} \geq \chi_{0.05}^{2}$, and as highly authentic if empirical value $\chi^{2} \geq \chi_{0.01}^{2}$. In the experiment, the number of participants in each session was limited by the number of computers in the classroom. Thus, for small groups (at $\mathrm{N}=15$, which is just our case) critical values $\chi^{2}$ are between $\chi_{0.05}^{2}=23,7$ to $\chi_{0.01}^{2}=29,1$ (see, e.g., [10]). The results of $\chi^{2}$-test are shown in Figure 3.

The main findings of the analysis are as follows:

1) the presence of statistically valid differences between "control" and "true" groups testifies to the possibility of registering the phenomenon of social inductance in the experiment performed;

2) in our experiment, there were no significant difference between the behaviour of "true" and "false" groups: the real manifestations of a social inductance are similar to those for mocked ones.

3) the experiment have shown the statistically valid increase of value $K$ for benefit of an answer which frequency of occurrence on the screen (be it in the "true" or "false" groups) was prevalent. 
Thus, a "strong" answer increased, a "weak" answer was suppressed. One can see this effect in the table of answers for the stimulus \#11 in Figure 1: starting from $\Delta_{0}=-0,15$ for the "control" group (for which the frequency of occurrence of the answer (a) is less than that of the answer (b)), under the influence of false hints $90 / 10$, the initially less probable answer prevails, $\Delta_{90 / 10}=+0,54$.

In Figure 4, dots, experimental values of ratio of "strong" to "weak" answers for all groups are shown. It is interesting that the deviation of numbers of answers from the average of distribution was observed much larger for "true" group than for all other groups ("control" and "false").

Let us remind that in the "true" group during all sessions and for all stimuli, the participants saw real answers of other participants of their groups.

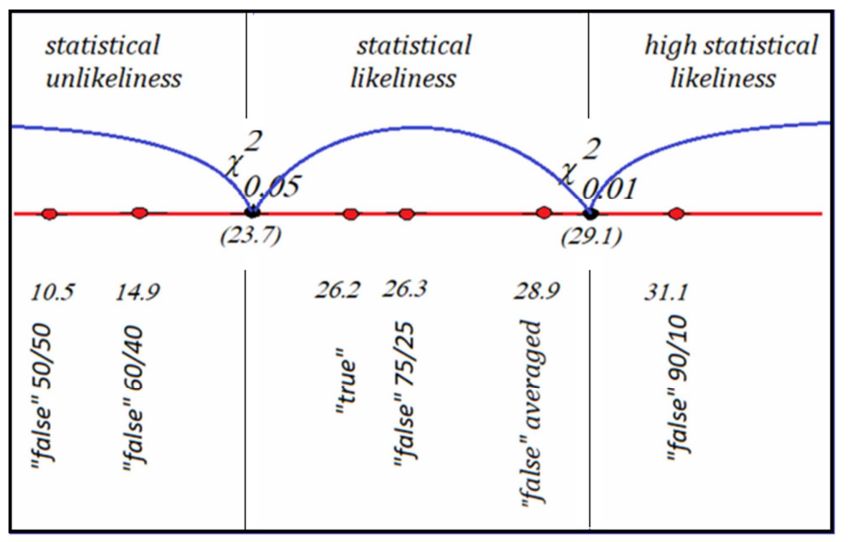

as compared to "control" group

Figure 3. Results of $\chi^{2-t e s t .}$

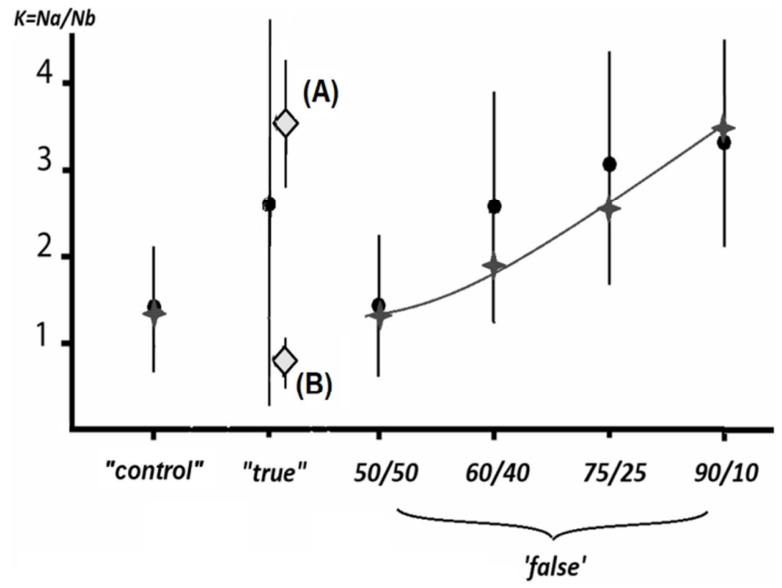

Figure 4. Experimental values of $K=N a / N b$ : dots - experimental values; curve-results of calculation for a laser-like model, see below.

An interpretation of this feature within our model will be given below.

\section{Laser-like Model and Discussion}

In the model, we consider that at the moment when participant is suggested to take part in the experiment, itsbrain is in a certain non-equilibrium state (upper level in Figure 5) analogous to an excited state of a quantum object (atom, molecule, ion...)

If participant agrees with this - the excitation is conserved on a kind of metastable level (Figure 5) until the start of the experiment, the relaxation is activated at the moment of appearance of a stimulus, and is realized by answering the question. Under conditions of choice between alternatives, the relaxation happens via two "channels" corresponding to answers (a) or (b).

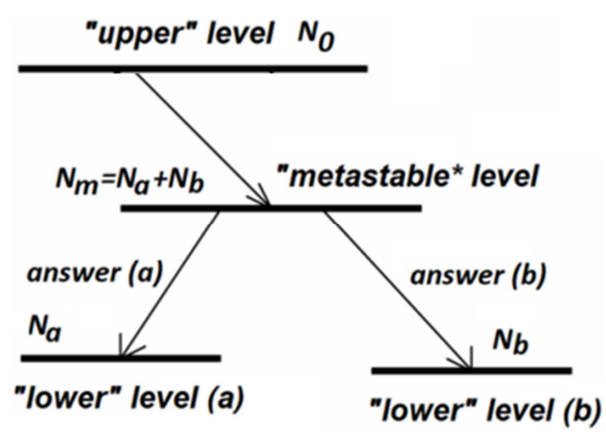

Figure 5. Laser-like scheme of states of brain.

This process has a certain likeliness to a spontaneous decay of excited state of an atom to two final states through two different channels.

The answers are registered by the computer and become known to other participants in a real ("true" group) or in a biased form ("false" groups).

Let us suppose that the answers that are sensed by other participants are capable to act similar to an external radiation caused stimulated emission.

The information about answers done by other participants is influencing the choice made by the recipient of this information, similar to hints and prompts in experiments of Asch [11] and other researchers [12-14].. Thus, one can plot a scheme (Figure 5) similar to schemes of quantum transitions from excited states, usual for laser studies, and to apply a corresponding mathematics.

Populations on different levels in this scheme can be described by a system of so called kinetic equations (see, e.g. [15]). For the case when prompting responses are visible to participants, for the "control" and "false" cases, this system can be written as follows:

$$
\begin{aligned}
\mathrm{dN}_{\mathrm{m}} / \mathrm{dt}=-\mathrm{N}_{\mathrm{m}}\left(\mathrm{A}_{\mathrm{a}}+\mathrm{A}_{\mathrm{b}}\right)-\mathrm{N}_{\mathrm{m}}\left(\mathrm{B}_{\mathrm{a}} \mathrm{W}_{\mathrm{a}}+\mathrm{B}_{\mathrm{b}} \mathrm{W}_{\mathrm{b}}\right)\left(\mathrm{N}_{\mathrm{a}}+\mathrm{N}_{\mathrm{b}}\right) \\
\mathrm{dN}_{\mathrm{a}} / \mathrm{dt}=\mathrm{N}_{\mathrm{m}} \mathrm{A}_{\mathrm{a}}+\mathrm{B}_{\mathrm{a}} \mathrm{W}_{\mathrm{a}} \mathrm{N}_{\mathrm{m}}\left(\mathrm{N}_{\mathrm{a}}+\mathrm{N}_{\mathrm{b}}\right) \\
\mathrm{dN}_{\mathrm{a}} / \mathrm{dt}=\mathrm{N}_{\mathrm{m}} \mathrm{A}_{\mathrm{b}}+\mathrm{B}_{\mathrm{b}} \mathrm{W}_{\mathrm{a}} \mathrm{N}_{\mathrm{m}}\left(\mathrm{N}_{\mathrm{a}}+\mathrm{N}_{\mathrm{b}}\right)
\end{aligned}
$$

Here: $N_{m}, N_{a}, N_{b}$ - populations of "metastable" and "lower" levels; $A_{a}, A_{b}-$ coefficients depicting frequencies of occurrence of answers (a) and (b) under conditions of individual choice (this is realized in the "control" group), similar to Einstein coefficients A for spontaneous decay of excited atoms; $B_{a}, B_{b}$ - analogues of Einstein coefficients B for induced transitions for channels (a) and (b), correspondingly; $W_{a}, W_{b}$ - coefficients which provide the frequency of occurrence for mocked answers (a) or (b) specified and predetermined by the experimenter. It is clear 
that for the "control" group one shall set $W_{a}=W_{b}=0$.

The initial conditions: $N_{m}(0)=N_{0} ; N_{a}(0)=N_{b}(0)=0$, In the equation (1) above the member $N_{m}\left(B_{a} W_{a}+B_{b} W_{b}\right)\left(N_{a}+N_{b}\right)$ describes the decay of "metastable" level under the influence

a)
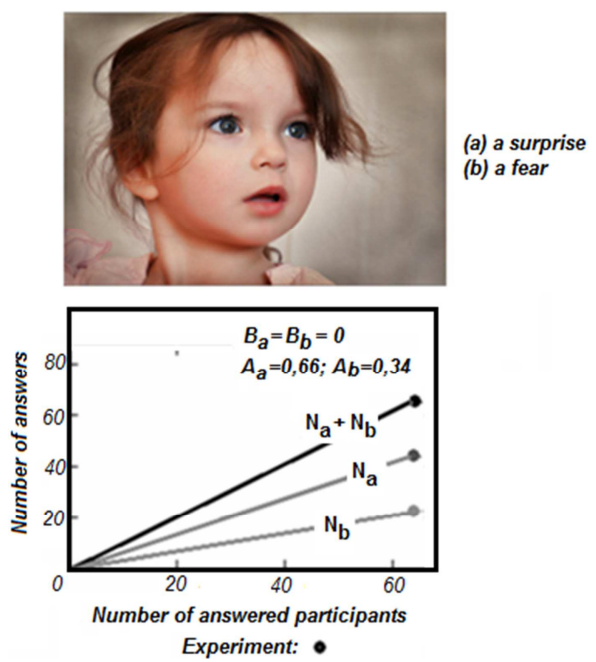

Figure 6. Example of calculation: a) - for the "control" group; b)-for all groups. of prompting responses, since $W_{a}\left(N_{a}+N_{b}\right)$ represents the number of generated false prompts for the answer (a); for the equations (2) and (3) similarly.

b)

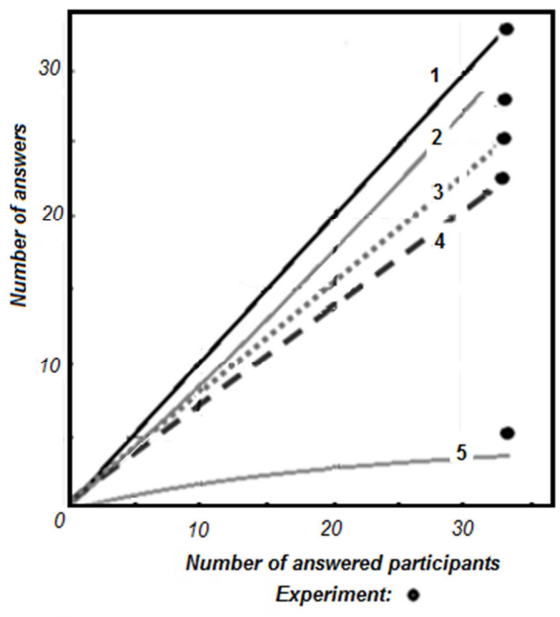

The example of calculation for one of stimuli for the "control" group is shown in Figure 6a).

By numerical calculations, one can fit values $B_{a}, B_{b}$ for getting the best agreement with experimental data. It was found that for all set of experimental data, all stimuli and all groups, the best agreement was for $B_{a}=6 \times 10^{-2}, B_{b}=4 \times 10^{-2}$.

For the case of "true" hints the equations (1-3) were modified as follows:

$$
\begin{gathered}
\mathrm{dN}_{\mathrm{m}} / \mathrm{dt}=-\mathrm{N}_{\mathrm{m}}\left(\mathrm{A}_{\mathrm{a}}+\mathrm{A}_{\mathrm{b}}\right)-\mathrm{N}_{\mathrm{m}}\left(\mathrm{B}_{\mathrm{a}} \mathrm{N}_{\mathrm{a}}+\mathrm{B}_{\mathrm{b}} \mathrm{N}_{\mathrm{b}}\right) \\
\mathrm{dN}_{\mathrm{a}} / \mathrm{dt}=\mathrm{N}_{\mathrm{m}} \mathrm{A}_{\mathrm{a}}+\mathrm{B}_{\mathrm{a}} \mathrm{N}_{\mathrm{a}} \\
\mathrm{dN}_{\mathrm{a}} / \mathrm{dt}=\mathrm{N}_{\mathrm{m}} \mathrm{A}_{\mathrm{b}}+\mathrm{B}_{\mathrm{b}} \mathrm{N}_{\mathrm{b}}
\end{gathered}
$$

These equations, in a fact, are standard kinetic equations for populations of levels in the presence of stimulated emission widely used in laser theory.

As an example, for the same stimulus as in Figure 6a, the results of experiment and calculations with the parameters $B_{a}$ and $B_{b}$ mentioned above are given in Figure $6 \mathrm{~b}$. In the figure: lines - calculation; dots - experimental data (total number of answers for the group); straight line 1 - sum of answers (a) and (b); curves 2-3 - values of $N_{a}$ for the "false 90/10" group (2) and the "true" group (3); curve $4-N_{a}$ for "control" group normalized for $N_{0}=34$; curve 5 - the value of $N_{b}$ for the "false 90/10" group.

The results of calculations of $K=N_{a} / N_{b}$ together with experimental data are presented in Figure 4 (see above). One can see a satisfactory correspondence between the results of calculation and experimental data.

Let us try to explain, in the frames of the model, the mentioned above feature of experimental results for the "true" group: much larger value of the deviation of answers from the average for this group than for all other groups. This can find its explanation in the difference of action of promoting responses.

For the "true" groups a random repeating set of first answers immediately provoke shift of subsequent answers to one of alternatives, thus operating as a positive feedback. At the same time, it is no such strong dependence of the final result to initial answers in the case of "false" groups; in the contrary, a constant relation $K=N_{a} / N_{b}$ set by the experimenter stabilizes final results against initial fluctuations of choice.

Taking initial conditions as $N_{a}(0)=6, N_{b}(0)=0$, one can get a theoretical solution indicated as $\mathrm{A}$ in Figure 4; taking them as $N_{a}(0)=0, N_{b}(0)=15$, we get the solution B in Figure 4. There was no monitoring of sequence of answers during the experiment, so one can suppose that such random initial conditions took place during the sessions, so the overall large deviation of results in the "true" case can be regarded as an illustration of high sensitivity of solutions of the system of equations (4-6) to initial conditions.

\section{Conclusion}

The scheme and results of our experiment gave some quantitative data concerning an influence of external opinions to the individual choice in conditions of uncertainty. These data allowed applying, to our experiment, a quantumlike model for energy levels in the brain and laser-like model of kinetic equations describing populations of excited and final states. Satisfactory results of comparison of theory and experiment give a hope to further development and productive application of laser-like models to problems of humans' social behavior thus answering the vital needs of society (see e.g. [16]).

Several immediate questions arise, for instance: 
1. How deep are analogies between lasers (as macroscopic objects with quantum behavior), from one hand, and human beings and a socium, from the other?

2. Analogies between effects of stimulated emission in physics, and an information influence to humans - to what extent are they universal?

3. What are boundaries of the analogy between human brain, in a state of decision making, and an atom having different energy states?

4. Are the determined values of coefficients of "stimulating influence" $B_{a}$ and $B_{b}$ applicable only for our experiment?

5. Will they vary with different sets of participants (ours were students; what for workers, office managers, artists, servicemen...)?

6. How to build and apply similar laser-like models for more vivid problems, as, e.g., choice between candidates at municipal elections?; etc.

It is clear that the described experiment had an artificial and separate character, and its immediate goal was to provide empirical background for justification a laser-like model. Surely, much wider research program shall be developed and fulfilled in order to reach an adequate description of psychological and social behavior; we believe that the presented results could help in this.

The material of the article has been reported at the IEEE International Conference «Video and Audio Signal Processing in the Context of Neurotechnologies» (SPCN), May 27-31,2019 at I. P. Pavlov's Inst. of Physiol., St.Petersburg, Russia, [17].

\section{References}

[1] A. Tversky, D Kahneman. (1983). Extensional versus intuitive reasoning: the conjunction fallacy in probability judgment. Psychol. Rev. 90: 293-315. doi: 10.1037/0033-295X.90.4.293.

[2] J. Busemeyer, E. Pothos, R. Franco, and J. Trueblood. (2011). A quantum theoretical explanation for probability judgment “errors". Psychol. Rev. 2: 193-218.

[3] F. Beck, J. Eccles. (2002.) Quantum aspects of brain activity and the role of consciousness. Proc Nat. Acad. Sci. USA. 23: 11357-61.

[4] A. Wendt. (2015). Quantum Mind and Social Science: Unifying Physical and Social Ontology. Cambridge: Cambridge University Press.
[5] E. Haven, A. Khrennikov. Quantum Social Science. New York: Cambridge University Press; 2013. p. 1-306.

[6] A. Zheltikov. (2018). The critique of quantum mind: measurement, consciousness, delayed choice, and lost coherence", UFN, 188: 10, 1119-1128; Phys. Usp., 61: 10 (2018), 1016-1025.

[7] N. Solovyev. (2019). Quantum neurophilosophy and the rehabilitation of the Cartesian model of consciousness. I. P. Pavlov Journ. of Higher Nervous Activity (Cited in PubMed as Zh. Vyss. Nerv. Deiat. I P Pavlova). St. Petersburg. 1: 120 29 (in Russian).

[8] O. Danilov, N. Rosanov, N. Solovyev, and L. Soms, (2016). Multimode lasers as analogs of complex biological systems (a survey). Opt. and Spectroscopy. 4: 646-53.

[9] A. Khrennikov, (2015). "Social Laser": Action amplification by stimulated emission of social Energy. arXiv: 1512.05386v1 [physics. soc-ph].

[10] Yu. Sidorenko. Methods of mathematical treatment in psychology. - St. Petersburg: Rech'; 2002. 1-350 (in Russian).

[11] S. Asch, (1956). Studies of independence and conformity. A minority of one against a unanimous majority. Psychol. Monographs. 9: 1-70.

[12] R. Crutchfield. (1955). Conformity and Character. American Psychologist, 10, 191-198. doi: 10.1037/h0040237.

[13] N. Hertz, E. Wiese. (2018). Under Pressure: Examining Social Conformity With Computer and Robot Groups. Human Factors: The Journal of the Human Factors and Ergonomics Society. 60 (8): 1207-1218. doi: 10.1177/0018720818788473.

[14] C. Kyrlitsias, M.-Grigoriou. (2018). Asch conformity experiment using immersive virtual reality. Computer Animation and Virtual Worlds. 29 (5); e1804. doi:. 10.1002/cav.1804.

[15] O. Svelto. Principles of Lasers. New York: Springer US; 2010. p. 255-63.

[16] A.. Sergeev A., «Social laser» and orange revolutions: an interview with President of RAS. http://лазер.pф/2019/01/11/10816/ (in Russian).

[17] D. Gorbatov, N. Solovyev, and L. Soms, "Social induction and a problem of choice in conditions of uncertainty", in: Neural Networks and Neurotechnologies. Yu. Shelepin, E. Ogorodnikova, N. Solovyev, E. Yakimova, Eds., St. Petersburg: Published by VVM, 2019, p. 116-128. 